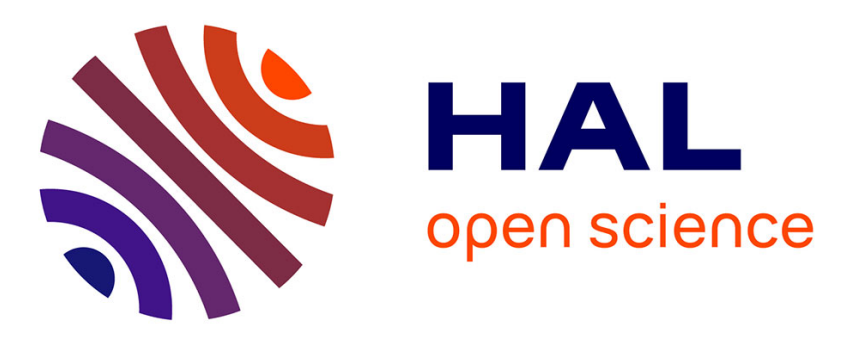

\title{
Force-Induced Dissolution of Imaginary Mode in Mechanochemical Reaction: Dibenzophenazine Synthesis
}

Naoki Haruta, Paulo Filho Marques De Oliveira, Tohru Sato, Kazuyoshi Tanaka, Michel Baron

\section{- To cite this version:}

Naoki Haruta, Paulo Filho Marques De Oliveira, Tohru Sato, Kazuyoshi Tanaka, Michel Baron. ForceInduced Dissolution of Imaginary Mode in Mechanochemical Reaction: Dibenzophenazine Synthesis. Journal of Physical Chemistry C, 2019, 123 (35), pp.21581-21587. 10.1021/acs.jpcc.9b05582 . hal02264273

\section{HAL Id: hal-02264273 \\ https://imt-mines-albi.hal.science/hal-02264273}

Submitted on 4 Oct 2019

HAL is a multi-disciplinary open access archive for the deposit and dissemination of scientific research documents, whether they are published or not. The documents may come from teaching and research institutions in France or abroad, or from public or private research centers.
L'archive ouverte pluridisciplinaire HAL, est destinée au dépôt et à la diffusion de documents scientifiques de niveau recherche, publiés ou non, émanant des établissements d'enseignement et de recherche français ou étrangers, des laboratoires publics ou privés. 


\title{
Force-Induced Dissolution of Imaginary Mode in Mechanochemical Reaction: Dibenzophenazine Synthesis
}

\author{
Naoki Haruta, ${ }^{\dagger \neq \odot ~ P a u l o ~ F i l h o ~ M a r q u e s ~ d e ~ O l i v e i r a, ~}{ }^{\|, \perp}$ Tohru Sato, ${ }^{*, \dagger, \neq, \S \odot ~ K a z u y o s h i ~ T a n a k a, ~}{ }^{\dagger, \ddagger}$ \\ and Michel Baron"
${ }^{\dagger}$ Fukui Institute for Fundamental Chemistry, Kyoto University, Takano-Nishihiraki-cho 34-4, Sakyo-ku, Kyoto 606-8103, Japan
${ }^{\ddagger}$ Department of Molecular Engineering, Graduate School of Engineering and ${ }^{\S}$ Unit of Elements Strategy Initiative for Catalysts \& Batteries, Kyoto University, Nishikyo-ku, Kyoto 615-8510, Japan
"Université de Toulouse, IMT Mines Albi, UMR CNRS 5302, Centre RAPSODEE, Campus Jarlard, 81013 Albi Cedex 09, France
${ }^{\perp}$ Institute of Chemistry, The University of São Paulo, Av. Prof. Lineu Prestes, 748, Vila Universitária, 05508-000 São Paulo, SP, \\ Brazil
}

\begin{abstract}
Dibenzophenazine was recently synthesized mechanochemically at a high yield. On the basis of experimental kinetics and theoretical vibronic coupling density analysis, this efficient synthesis has been expected to involve two types of reaction mechanisms. In this study, the reaction pathways, including the catalytic effect of water, are determined using density functional theory. The lowest-energy path involves stepwise formation of two $\mathrm{C}-\mathrm{N}$ bonds, and a concerted path with a higher barrier is also found. The highest-energy point along the latter path is not a transition state but a higher-order saddle point. Therefore, the concerted path is not generally accepted as a reaction path. However, even a small mechanical force dissolves imaginary frequencies so that the higher-order saddle point is changed to a transition state. This mechanical dissolution of imaginary frequencies is a new feature of theoretical mechanochemistry.

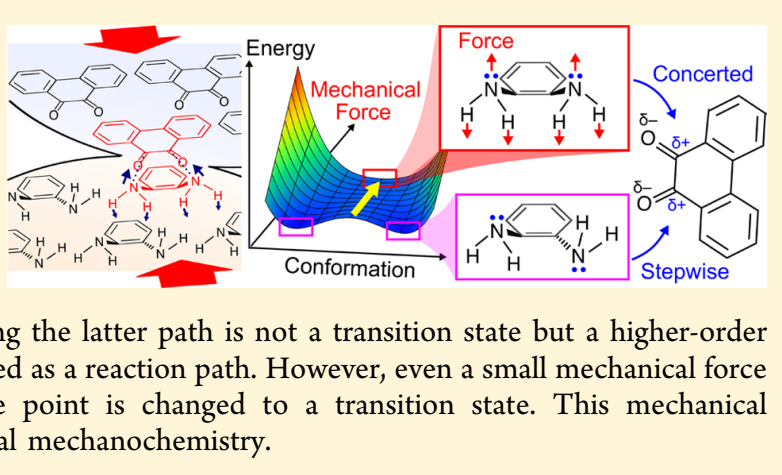

\section{INTRODUCTION}

Mechanochemistry ${ }^{1,2}$ is the study of chemical phenomena using mechanical actions such as grinding. In particular, mechanochemistry related to friction is well known as tribochemistry. ${ }^{3-5}$ Friction sometimes causes mechanical excitation on the order of $\mathrm{eV}$, leading to luminescence called triboluminescence. ${ }^{6}$ Such mechanical excitation can also drive chemical reactions. Mechanochemical reactions have attracted much attention recently because of their eco-friendliness as no solvent is needed. In conventional reactions, thermal excitation is a driving force that overcomes reaction barriers. However, mechanochemical reactions are not always conventional thermal reactions. Mechanochemical conditions sometimes cause unexpected chemical reactions. For example, Hickenboth et al. have reported that the Woodward-Hoffmann rule ${ }^{7}$ can be broken in mechanochemistry. ${ }^{8}$ To explain these unique features of mechanochemistry, Ribas-Arino et al. have proposed that mechanical force modifies the potential energy surface and changes the reaction barriers. ${ }^{9,10}$

One typical example of mechanochemical reactions is the synthesis of dibenzophenazine (DBPZ), a well-known quinoxaline-containing molecule (see Figure 1). The quinoxaline structure is an important building block of organic semiconductors and pharmacologically active compounds. ${ }^{11} \mathrm{DBPZ}$ has been synthesized in various ways. ${ }^{12-50}$ Conventional syntheses require both a solvent and a catalyst. More

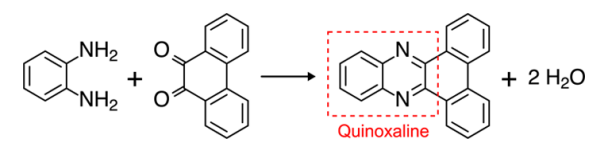

Figure 1. Synthetic scheme of dibenzophenazine (DBPZ) from $o$ phenylenediamine (PDA) and 9,10-phenanthrenequinone (PQ). DBPZ has a quinoxaline structure.

specifically, Brønsted acids, ${ }^{12,15,16,18,22,26,28,30,33,44}$ metal oxide nanoparticles, ${ }^{16,18,19,22,25,27,28,30,36,42,43}$ and ionic liquids ${ }^{17,37,38,40,41,46}$ have been used. Recently, however, Carlier et al. have synthesized DBPZ using only mechanical ball-milling ${ }^{20}$ without any solvent or catalyst and have obtained a very high yield $(>99 \%)$.

To investigate the efficient mechanochemical synthesis of DBPZ, experimental and theoretical studies have been reported. ${ }^{51-53}$ The yield obtained $24 \mathrm{~h}$ after mechanical grinding is greater than that obtained immediately after grinding. ${ }^{51}$ That is, the product is generated even after grinding is complete. The yield obtained immediately after grinding is proportional to the grinding time, whereas the yield obtained $24 \mathrm{~h}$ after grinding is not. In addition, the reaction 
heat flow has two peaks, that is, early and delayed ones. ${ }^{52}$ These experimental facts suggest the possibility of different reaction mechanisms.

In our previous work, ${ }^{53} \mathrm{DBPZ}$ synthesis was theoretically investigated using vibronic coupling density (VCD) analysis. $^{54,55}$ One of the reactants, $o$-phenylenediamine (PDA), has a high-lying highest occupied molecular orbital (HOMO). Another reactant, 9,10-phenanthrenequinone (PQ), has a lowlying lowest unoccupied molecular orbital (LUMO). According to the frontier orbital theory, ${ }^{56,57}$ the regioselectivity should be indicated by these frontier orbitals. However, the HOMO of PDA is strongly delocalized over the molecule. Thus, the frontier orbital theory fails to predict its regioselectivity.

The frontier orbital theory takes into account only stabilization due to charge-transfer (orbital) interaction and does not consider any molecular deformation. In contrast, the VCD theory takes into account stabilization due to molecular deformation. The VCD can be regarded as a reactivity index in the framework of the conceptual density functional theory (DFT) ${ }^{58,59}$ VCD analysis has identified reactive regions in fullerenes, nanographenes, and so on, for which the frontier orbital theory is not effective. ${ }^{53-55,60-66}$ We have applied VCD analysis to PDA and PQ and successfully identified their reactive sites.

PDA has two conformers with $C_{2}$ and $C_{s}$ symmetries depending on the conformation of its two pyramidal amino groups. $C_{2} \mathrm{PDA}$ is the most stable one, whereas $C_{s} \mathrm{PDA}$ is unstable with one imaginary frequency. According to VCD analysis, $C_{2}$ PDA favors stepwise formation of $\mathrm{C}-\mathrm{N}$ bonds between the two reactants, whereas $C_{s}$ PDA favors concerted formation. Because the mechanical force must increase the amount of $C_{s}$ PDA during grinding, the concerted reaction is considered to be possible under mechanochemical conditions.

Although VCD analysis predicts both stepwise and concerted mechanisms, these reaction pathways and their energy barriers, which are indispensable for quantitative and even qualitative discussion, have not been determined to date. Our goal in this study is to identify all the transition states and their reaction barriers and then propose reaction pathways under mechanochemical conditions from a theoretical viewpoint. In addition, more importantly, a novel concept of mechanical modification of a reaction pathway is also proposed: force-induced dissolution of imaginary frequencies.

\section{COMPUTATIONAL DETAILS}

We performed geometry optimizations and vibrational analyses of all the reactants, intermediates, transition states, and products using DFT calculations. We calculated the intrinsic reaction coordinates to confirm the validity of the obtained transition states. We also calculated the internal energies, enthalpies, and Gibbs free energies of all the species at 298.15 $\mathrm{K}$ and $1.0000 \mathrm{~atm}$ to examine the thermochemistry. All the energies presented in this paper include zero-point vibrational energy corrections. These DFT calculations were performed at the B3LYP/6-311G $(\mathrm{d}, \mathrm{p})$ level of theory using the Gaussian 09 program. ${ }^{67}$

\section{RESULTS AND DISCUSSION}

3.1. Reaction Pathways. DBPZ synthesis consists of two $\mathrm{C}-\mathrm{N}$ bond formations and two dehydration processes. Figure 2 shows the obtained energy diagram of the reaction pathways

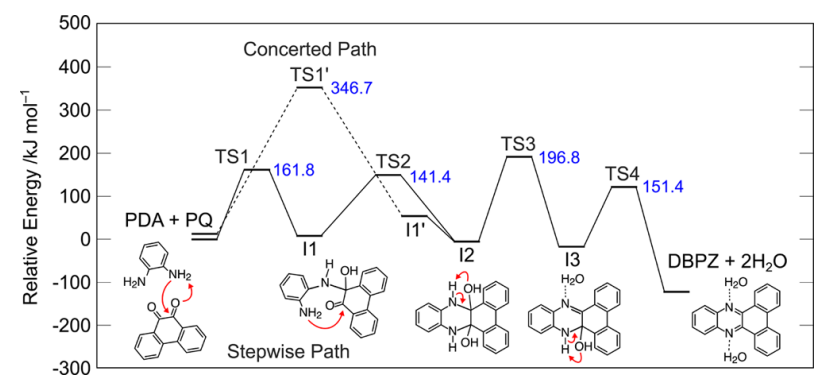

Figure 2. Energy diagram with two reaction pathways. Values in blue adjacent to transition states indicate activation energies in $\mathrm{kJ} \mathrm{mol}^{-1}$. Initial species have different energies because PDA has different conformers.

and their activation energies. The structures of the reactants and products, intermediates, and transition states are shown in Figures 3-5, respectively. The lowest-energy reaction path was
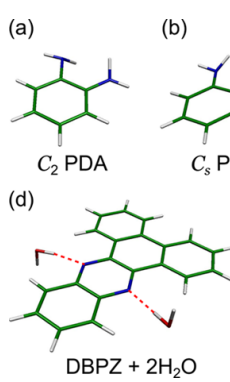

(b)

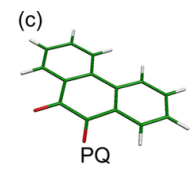

(e)

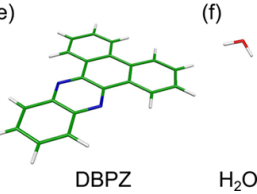

Figure 3. Optimized structures of (a) $C_{2}$ PDA, (b) $C_{s} P D A,(c) P Q$ (d) DBPZ coordinated by two water molecules, (e) DBPZ, and (f) a water molecule. The red dashed lines indicate hydrogen bonds.

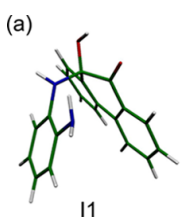

(c)

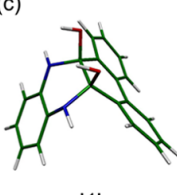

(b)

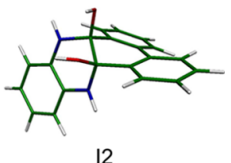

(d)

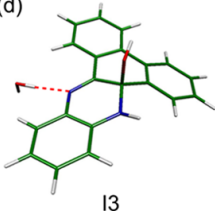

Figure 4. Optimized structures of (a) I1, (b) I2, (c) I1' ${ }^{\prime}$, and (d) I3. I1 ${ }^{\prime}$ has a structure similar to that of $\mathbf{I 2}$ and $C_{s}$ symmetry with one imaginary frequency. The red dashed line indicates a hydrogen bond.

found to be the stepwise path, which involves successive formation of two $\mathrm{C}-\mathrm{N}$ bonds. In the stepwise path, both the first and second $\mathrm{C}-\mathrm{N}$ bond formations have almost the same high reaction barriers: 161.8 and $141.4 \mathrm{~kJ} \mathrm{~mol}^{-1}$, respectively. Furthermore, the subsequent dehydration process requires higher energy than these $\mathrm{C}-\mathrm{N}$ bond formations. Thus, the first dehydration process seems to be the rate-determining step. Note, however, that a water molecule produced in the same or a neighboring system catalyzes both the first and second dehydration processes, as discussed later. The catalytic water greatly reduces the activation energies. 
TS1

(d)

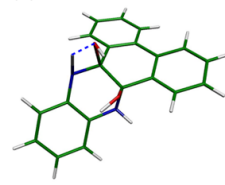

TS3

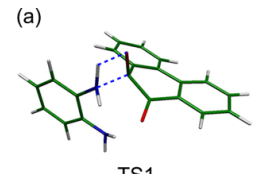

(b)

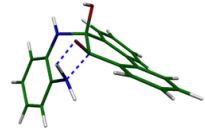

TS2

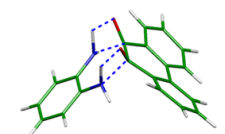

TS $1^{\prime}$

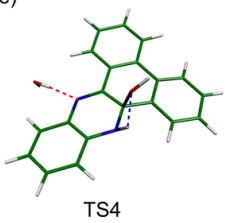

Figure 5. Optimized structures of (a) TS1, (b) TS2, (c) TS1', (d) TS3, and (e) TS4. The red dashed line indicates a hydrogen bond. Note that TS1' has $C_{s}$ symmetry with the imaginary frequencies of $a^{\prime \prime}$ modes as well as the $a^{\prime}$ reaction mode.

In addition to the stepwise path, a concerted one was also identified; it involves simultaneous formation of two $\mathrm{C}-\mathrm{N}$ bonds. In the concerted path, the formation of two $\mathrm{C}-\mathrm{N}$ bonds has the highest energy barrier. Because the subsequent dehydration processes have much lower barriers, they are considered to occur rapidly. Although the concerted path has a higher barrier than the stepwise path, the difference is only $184.9 \mathrm{~kJ} \mathrm{~mol}^{-1}$, which can be overcome by mechanical excitation (on the order of $\mathrm{eV})^{6}$ but not by thermal excitation.

Regarding the concerted path, we should note that PDA, TS1', and I2' have $C_{s}$ symmetry with the imaginary frequencies of $a^{\prime \prime}$ modes, which are symmetry-lowering modes. Thus, the concerted path is not generally accepted as a reaction path (see Figure 6) because a transition state should

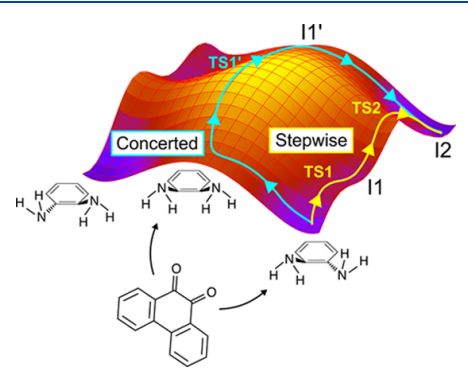

Figure 6. Schematic representation of stepwise and concerted pathways on potential energy surface. A concerted path with multiple imaginary frequencies is not generally accepted as a reaction path. However, an external force can dissolve imaginary frequencies.

have only one imaginary frequency by definition. Later, we show that the instability of this path can be resolved by applying an external mechanical force.

Table 1 shows the calculated heat of reaction. This reaction is exothermic and produces a large amount of heat because of aromatization and hydrogen-bond formation between DBPZ and two water molecules (see Figure 3d). These hydrogen bonds stabilize the final product by $51.4 \mathrm{~kJ} \mathrm{~mol}^{-1}$. The energy of hydrogen-bond formation was found to account for $\sim 40 \%$ of the overall heat of reaction. The rest can be attributed mainly to aromatization.

3.2. Catalytic Effect of Water. Hydrogen bonds can be broken under mechanochemical conditions because the bond energies are smaller than the mechanical energy. The free water molecules interact with reaction centers such as $-\mathrm{OH}$ and $-\mathrm{NH}-$ groups and catalyze the dehydration steps. An
Table 1. Obtained Heat of Reaction ${ }^{a}$

\begin{tabular}{lcccc}
\multicolumn{1}{c}{ factor } & $\begin{array}{c}\Delta E \\
\left(\mathrm{~kJ} \mathrm{~mol}^{-1}\right)\end{array}$ & $\begin{array}{c}\Delta U \\
\left(\mathrm{~kJ} \mathrm{~mol}^{-1}\right)\end{array}$ & $\begin{array}{c}\Delta H \\
\left(\mathrm{~kJ} \mathrm{~mol}^{-1}\right)\end{array}$ & $\begin{array}{c}\Delta G \\
\left(\mathrm{~kJ} \mathrm{~mol}^{-1}\right)\end{array}$ \\
$\begin{array}{c}\text { two hydrogen } \\
\text { bonds }\end{array}$ & -51.4 & -49.1 & -54.1 & 18.7 \\
aromatization & -70.3 & -65.3 & -62.8 & -92.9 \\
overall & -121.7 & -114.4 & -116.9 & -74.2
\end{tabular}

${ }^{a} \Delta E, \Delta U, \Delta H$, and $\Delta G$ denote the change in electronic and nuclearnuclear repulsive energies, change in internal energy, change in enthalpy, and change in Gibbs free energy, respectively, at $298.15 \mathrm{~K}$ and 1.0000 atm.

energy diagram including the catalytic effect of water is shown in Figure 7. The structures of the related species are illustrated

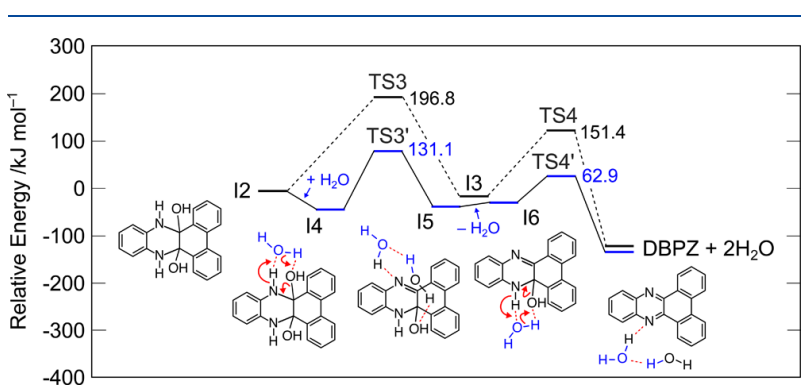

Figure 7. Energy diagram showing the catalytic effect of water. The energy of an additional water molecule was properly subtracted. Although water catalysis requires breaking of a hydrogen bond, the bond energy is sufficiently small $\left(\sim 25.7 \mathrm{~kJ} \mathrm{~mol}^{-1}\right)$, as shown in Table 1. Values adjacent to transition states indicate activation energies in $\mathrm{kJ}$ $\mathrm{mol}^{-1}$. (a)

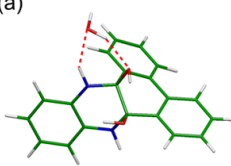

14

(d)

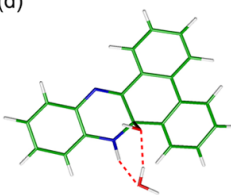

16

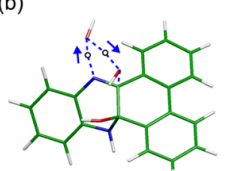

TS3

(e)

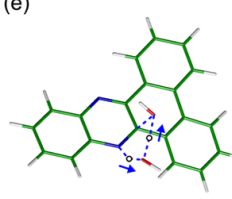

TS4' (c)
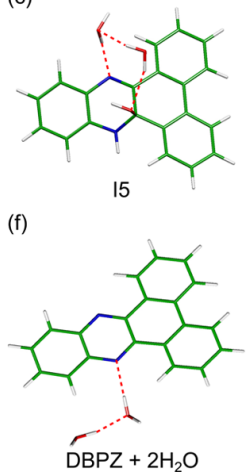

Figure 8. Optimized structures of (a) I4, (b) TS3', (c) I5, (d) I6, (e) TS4', and (f) DBPZ with two water molecules produced by water catalysis. The red dashed lines indicate hydrogen bonds.

in Figure 8. The catalytic water lowers the activation energies of the dehydration steps by $60-90 \mathrm{~kJ} \mathrm{~mol}^{-1}$. Therefore, neither dehydration step is the rate-determining step. In DBPZ synthesis, the formation of the two $\mathrm{C}-\mathrm{N}$ bonds is rather important for its kinetics.

On the basis of the obtained results, we discuss plausible reaction mechanisms under mechanochemical conditions. All the reaction barriers are too high to be overcome by thermal energy. This reaction is therefore difficult under conventional thermal condition without any catalyst. This is consistent with many experimental reports. ${ }^{12-50}$ However, under mechano- 
chemical conditions, mechanical action can generate sufficient energy (on the order of $\mathrm{eV}$ ) locally. ${ }^{6}$ Therefore, both the stepwise and concerted paths are possible during grinding. After grinding, the energy difference between the two paths cannot be ignored. The stepwise path, with its lower barriers, must be favored. However, even these barriers are too high. In solid-state reactions, energy transfer can occur among neighboring reactants, intermediates, and products. Therefore, the supplied mechanical energy and large heat of reaction can be reused to conduct the reaction successively. This is in contrast to reactions in a solution, in which solvents separate reactant molecules from each other. We recently reported a detailed analysis of the experimentally observed reaction heat flow and kinetics that supports the findings of the present study. ${ }^{52}$

3.3. Removal of Instability by External Force. The obtained concerted path has the imaginary frequencies of the $a^{\prime \prime}$ modes, which are symmetry-lowering modes. They originate from the instability of $C_{s}$ PDA (see Figure 9a). Consequently, the concerted path is not generally accepted as a reaction path. We are interested in whether the instability is conserved under mechanical conditions. (a)

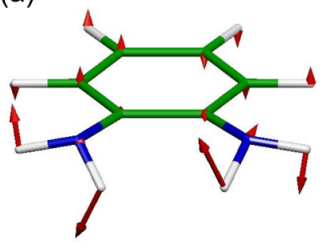

(b)

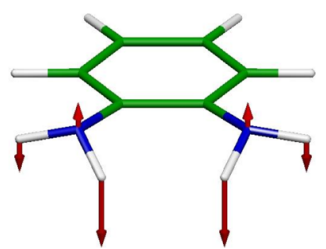

Figure 9. (a) $a^{\prime \prime}(1)$ normal mode of $C_{s}$ PDA with an imaginary frequency of $252 i \mathrm{~cm}^{-1}$. (b) Totally symmetric deformation mode with the planarization of the pyramidal amino groups in the opposite direction.

During mechanical grinding, a random external force is applied to each reactant inside microcrystalline grains or at the interface of the grains. We should herein pay attention to whether an external force removing the instability of the concerted path is possible or not. If possible, such an external force can be applied at a certain moment, thereby opening the concerted path. At other moments, an external force might be useless for opening the concerted path, and hence the stepwise path is preferred.

Application of an external mechanical force is theoretically equivalent to deformation of the equilibrium structure. A molecular deformation corresponding to an external force reportedly modifies the potential energy surface and changes the reaction barriers. ${ }^{9,10}$ Mathematically, this can be understood as the Legendre transformation of the potential energy surface as position $\mathbf{r} \rightarrow$ force $\mathbf{f}$.

To simulate application of a mechanical force to PDA, we considered a deformation of $C_{s}$ PDA defined as a change in the planarization of the pyramidal amino groups to the opposite direction (see Figure 9b). This deformation is described in detail in the Supporting Information. This mode is orthogonal to the $a^{\prime \prime}$ mode with the imaginary frequency because it has $a^{\prime}$ symmetry. As shown in Table 2, we found that the imaginary frequency of the $a^{\prime \prime}$ mode becomes real when $C_{s}$ PDA is deformed by this $a^{\prime}$ mode. Note that even if the $a^{\prime}$ deformation is applied, the force along the $a^{\prime \prime}$ mode is still zero because of
Table 2. PDAs Deformed by the Totally Symmetric Deformation Mode and the Frequencies of the $a^{\prime \prime}(1)$ Normal Mode ${ }^{a}$

\begin{tabular}{|c|c|c|c|c|c|}
\hline $\begin{array}{l}\text { totally symmetric deformation } \\
\qquad\left(10^{-2} \AA\right)\end{array}$ & 0 & 1 & 2 & 3 & 4 \\
\hline frequency of $a^{\prime \prime}(1)$ mode $\left(\mathrm{cm}^{-1}\right)$ & $250 i$ & $167 i$ & 50 & 148 & 166 \\
\hline
\end{tabular}

its symmetry. That is, the deformed $C_{s}$ PDA is always fully optimized with respect to the $a^{\prime \prime}$ mode.

The strength of the applied force can be estimated using a potential gradient $\partial E / \partial Q_{d}$, where $E$ denotes the total electronic energy with the nuclear-nuclear potentials, and $Q_{d}$ is a massweighted coordinate of the $a^{\prime}$ deformation mode. As shown in Figure 10a, the strength of the force is proportional to the
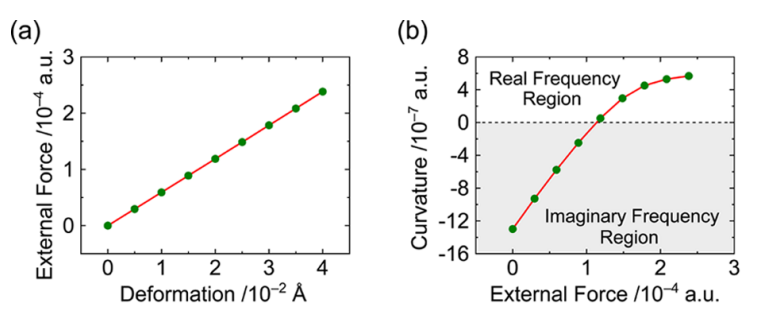

(c)

(d) Mechanical Force
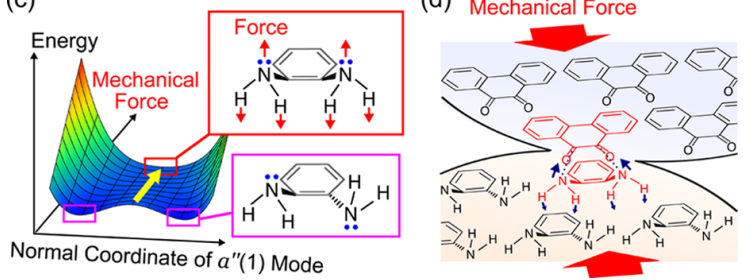

Figure 10. (a) Relation between the totally symmetric mechanical force acting on $C_{s}$ PDA and its molecular deformation. (b) Curvature $\partial^{2} E / \partial Q^{2}$ of the $a^{\prime \prime}(1)$ mode as a function of the mechanical force. (c) Conceptual illustration of potential energy surface as a function of a normal coordinate of the $a^{\prime \prime}(1)$ mode and the mechanical force. (d) Illustration of interfacial stacking of microcrystalline grains of PDA and PQ under mechanical pressure.

deformation. Figure $10 \mathrm{~b}$ shows that increasing the force makes the curvature of the $a^{\prime \prime}$ mode positive. The positive curvature is equivalent to a real frequency. A conceptual illustration is shown in Figure 10c.

This result shows that if $C_{s}$ PDA is mechanically deformed by the $a^{\prime}$ deformation mode, then the $a^{\prime \prime}$ instability in the concerted path is resolved. That is, the mechanical action leads to a new reaction path. In practical terms, this effect could be attributed to the interfacial stacking of microcrystalline grains of PDA and PQ under mechanical pressure, as illustrated in Figure 10d. This effect is not possible under conventional thermal conditions. The mechanical force necessary to remove the instability is small $\left(\sim 10^{-4}\right.$ a.u.). Accordingly, it does not significantly change the obtained energy barriers of the concerted path.

3.4. Origin of Instability Removal. To elucidate the origin of the force-induced dissolution of the imaginary mode, a simple model potential was constructed, which depends only on two mass-weighted normal coordinates, $Q_{\alpha}$ and $Q_{\beta}$. In this study, the two coordinates can be regarded as the normal coordinate of the $a^{\prime \prime}$ imaginary mode and $a^{\prime}$ deformation coordinate, respectively. The model potential $E$ is given by 

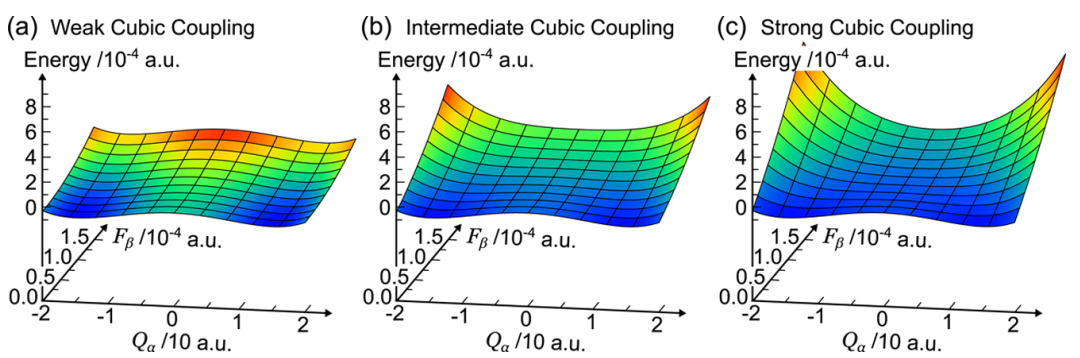

Figure 11. Model potentials determined using the obtained fitting parameters. The values of the cubic force constant $\gamma$ were (a) 0 , (b) 1 , and (c) 2 times the DFT-based value.

$$
E=E_{\alpha}\left(Q_{\alpha}\right)+E_{\beta}\left(Q_{\beta}\right)+E_{\alpha \beta}\left(Q_{\alpha}, Q_{\beta}\right)
$$

The frequency of mode $\alpha$ is imaginary, and thus, $E_{\alpha}$ is a double minimum potential

$$
E_{\alpha}\left(Q_{\alpha}\right)=\frac{1}{2} \omega_{\alpha}{ }^{2} Q_{\alpha}{ }^{2}+\frac{1}{4 !} \delta_{\alpha} Q_{\alpha}{ }^{4} \quad\left(\omega_{\alpha}{ }^{2}<0, \delta_{\alpha}>0\right)
$$

where $\omega_{\alpha}$ denotes the frequency of mode $\alpha$, and $\delta_{\alpha}$ is a quartic force constant. By contrast, the frequency of mode $\beta$ is real, and thus, $E_{\beta}$ is a single minimum potential

$$
E_{\beta}\left(Q_{\beta}\right)=\frac{1}{2} \omega_{\beta}^{2} Q_{\beta}^{2} \quad\left(\omega_{\beta}^{2}>0\right)
$$

where $\omega_{\beta}$ is the frequency of mode $\beta$. Furthermore, a cubic force constant $\gamma$ was considered as the intermode coupling

$$
E_{\alpha \beta}\left(Q_{\alpha}, Q_{\beta}\right)=\frac{1}{2} \gamma Q_{\alpha}{ }^{2} Q_{\beta}
$$

Application of an external mechanical force shifts the equilibrium point. Applying a deformation $Q_{\beta}=\Delta$ is equivalent to applying the force given by

$$
F_{\beta}=\left(\frac{\partial E}{\partial Q_{\beta}}\right)_{Q_{\alpha}=0, Q_{\beta}=\Delta}=\omega_{\beta}^{2} \Delta
$$

The potential curve under the condition $Q_{\beta}=\Delta$ is obtained as

$$
\begin{aligned}
E & =E_{\alpha}\left(Q_{\alpha}\right)+E_{\beta}(\Delta)+E_{\alpha \beta}\left(Q_{\alpha}, \Delta\right) \\
& =\frac{1}{2}\left(\omega_{\alpha}{ }^{2}+\gamma \Delta\right) Q_{\alpha}{ }^{2}+\frac{1}{4 !} \delta_{\alpha} Q_{\alpha}{ }^{4}+\frac{1}{2} \omega_{\beta}{ }^{2} \Delta^{2}
\end{aligned}
$$

Consequently, the curvature of mode $\alpha$ depends on $F_{\beta}$ owing to the cubic force constant

$$
\left(\frac{\partial^{2} E}{\partial Q_{\alpha}{ }^{2}}\right)_{Q_{\alpha}=0, Q_{\beta}=\Delta}=\omega_{\alpha}^{2}+\gamma \Delta=\omega_{\alpha}^{2}+\frac{\gamma}{\omega_{\beta}{ }^{2}} F_{\beta}
$$

The DFT-based potential surface of PDA was numerically fitted by the model potential, and the fitting parameters were determined as follows: $\omega_{\alpha}=1.14 i \times 10^{-3}$ a.u., $\delta_{\alpha}=3.47 \times 10^{-8}$ a.u., $\omega_{\beta}=7.74 \times 10^{-3}$ a.u., and $\gamma=6.73 \times 10^{-7}$ a.u. Figure 11 shows the model potentials obtained using these parameters. The values of the cubic force constant $\gamma$ were 0,1 , and 2 times the DFT-based value, which correspond to weak, intermediate, and strong coupling, respectively. The obtained result clearly illustrates that the cubic force constant is essential for the force-induced dissolution of the imaginary mode.

\section{CONCLUSIONS}

The reaction pathways in mechanochemical synthesis of DBPZ were investigated using DFT. According to our computational results, the minimum-energy path involves stepwise $\mathrm{C}-\mathrm{N}$ bond formations between two reactants. In addition, a concerted path with a higher energy barrier was also found. The latter is generally not accepted as a reaction path because the highest-energy point along the path is not a transition state but a higher-order saddle point. However, even a small mechanical force dissolves the imaginary frequencies so that the higher-order saddle point is changed into a transition state. The mechanical force identified here is one example of how to make the imaginary frequencies real. To find such forces in a mechanochemical reaction may become a central problem in theoretical mechanochemistry.

\section{AUTHOR INFORMATION}

\section{Corresponding Author}

*E-mail: tsato@scl.kyoto-u.ac.jp. Phone: +81-75-711-7849. Fax: +81-75-711-7849.

\section{ORCID $\odot$}

Naoki Haruta: 0000-0002-6635-1570

Tohru Sato: 0000-0002-4554-8411

\section{Notes}

The authors declare no competing financial interest.

\section{ACKNOWLEDGMENTS}

This research was supported by the JSPS-CNRS bilateral program. The Embassy of France (Exploration Japon 2017) is also gratefully acknowledged. Parts of the numerical calculations were performed at the Supercomputer Laboratory of Kyoto University and the Research Center for Computational Science, Okazaki, Japan. We would like to thank Editage (www.editage.jp) for English language editing.

\section{REFERENCES}

(1) Baron, M. Towards a Greener Pharmacy by More Eco Design. Waste Biomass Valorization 2012, 3, 395-407. 
(2) Wang, G.-W. Mechanochemical Organic Synthesis. Chem. Soc. Rev. 2013, 42, 7668-7700.

(3) Hsu, S. M.; Zhang, J.; Yin, Z. The Nature and Origin of Tribochemistry. Tribol. Lett. 2002, 13, 131-139.

(4) Boldyreva, E. Mechanochemistry of Inorganic and Organic Systems: What Is Similar, What Is Different? Chem. Soc. Rev. 2013, 42, 7719-7738.

(5) Tysoe, W. On Stress-Induced Tribochemical Reaction Rates. Tribol. Lett. 2017, 65, 48.

(6) Zink, J. I. Triboluminescence. Acc. Chem. Res. 1978, 11, 289295.

(7) Woodward, R. B.; Hoffmann, R. The Conservation of Orbital Symmetry. Angew. Chem., Int. Ed. Engl. 1969, 8, 781-853.

(8) Hickenboth, C. R.; Moore, J. S.; White, S. R.; Sottos, N. R.; Baudry, J.; Wilson, S. R. Biasing Reaction Pathways with Mechanical Force. Nature 2007, 446, 423-427.

(9) Ribas-Arino, J.; Shiga, M.; Marx, D. Understanding Covalent Mechanochemistry. Angew. Chem., Int. Ed. 2009, 48, 4190-4193.

(10) Ribas-Arino, J.; Marx, D. Covalent Mechanochemistry: Theoretical Concepts and Computational Tools with Applications to Molecular Nanomechanics. Chem. Rev. 2012, 112, 5412-5487.

(11) Martins, M. A. P.; Frizzo, C. P.; Moreira, D. N.; Buriol, L.; Machado, P. Solvent-Free Heterocyclic Synthesis. Chem. Rev. 2009, 109, 4140-4182.

(12) Minsky, A.; Cohen, Y.; Rabinovitz, M. Novel Polycyclic Dianions: Metal Reduction of Nitrogen Heterocycles. J. Am. Chem. Soc. 1985, 107, 1501-1505.

(13) Kumamoto, K.; Iida, H.; Hamana, H.; Kotsuki, H.; Matsumoto, K. Are Multicomponent Strecker Reactions of Diketones with Diamines Under High Pressure Amenable to Heterocyclic Synthesis? Heterocycles 2005, 66, 675-681.

(14) Farus, O. A.; Balashev, K. P.; Ivanov, M. A.; Tkacheva, T. A.; Panova, A. G. Preparation and Spectroscopic and Electrochemical Properties of Complexes of dibenzo-and Dipyrido-substituted 1,4Diazines. Russ. J. Gen. Chem. 2006, 76, 311-316.

(15) Niknam, K.; Zolfigol, M. A.; Tavakoli, Z.; Heydari, Z. Metal Hydrogen Sulfates $\mathrm{M}\left(\mathrm{HSO}_{4}\right)_{\mathrm{n}}$ : As Efficient Catalysts for the Synthesis of Quinoxalines in EtOH at Room Temperature. J. Chin. Chem. Soc. 2008, 55, 1373-1378.

(16) Niknam, K.; Saberi, D.; Mohagheghnejad, M. Silica Bonded SSulfonic Acid: A Recyclable Catalyst for the Synthesis of Quinoxalines at Room Temperature. Molecules 2009, 14, 1915-1926.

(17) Zare, A.; Hasaninejad, A.; Parhami, A.; Moosavi-Zare, A. R.; Khedri, F.; Parsaee, Z.; Abdolalipoor-Saretoli, M.; Khedri, M.; Roshankar, M.; Deisi, H. Ionic Liquid 1-Butyl-3-methylimidazolium Bromide ([bmim $] \mathrm{Br})$ : A Green and Neutral Reaction Media for the Efficient, Catalyst-free Synthesis of Quinoxaline Derivatives. J. Serb. Chem. Soc. 2010, 75, 1315-1324.

(18) Rashedian, F.; Saberi, D.; Niknam, K. Silica-Bonded N-Propyl Sulfamic Acid: A Recyclable Catalyst for the Synthesis of 1,8-Dioxodecahydroacridines, 1,8-Dioxo-octahydroxanthenes and Quinoxalines. J. Chin. Chem. Soc. 2010, 57, 998-1006.

(19) Sharma, R. K.; Sharma, C. Zirconium(IV)-modified Silica Gel: Preparation, Characterization and Catalytic Activity in the Synthesis of Some Biologically Important Molecules. Catal. Commun. 2011, 12, $327-331$.

(20) Carlier, L.; Baron, M.; Chamayou, A.; Couarraze, G. Use of Cogrinding as a Solvent-free Solid State Method to Synthesize Dibenzophenazines. Tetrahedron Lett. 2011, 52, 4686-4689.

(21) Chavan, H. V.; Adsul, L. K.; Bandgar, B. P. Polyethylene Glycol in Water: A Simple, Efficient and Green Protocol for the Synthesis of Quinoxalines. J. Chem. Sci. 2011, 123, 477-483.

(22) Karami, B.; Khodabakhshi, S.; Nikrooz, M. Synthesis of AzaPolycyclic Compounds: Novel Phenazines and Quinoxalines Using Molybdate Sulfuric Acid (MSA). Polycyclic Aromat. Compd. 2011, 31, 97-109.

(23) Bachhav, H. M.; Bhagat, S. B.; Telvekar, V. N. Efficient Protocol for the Synthesis of Quinoxaline, Benzoxazole and
Benzimidazole Derivatives Using Glycerol as Green Solvent. Tetrahedron Lett. 2011, 52, 5697-5701.

(24) Karami, B.; Khodabakhshi, S. A Facile Synthesis of Phenazine and Quinoxaline (new 1,4-benzo diazine) Derivatives Using Magnesium Sulfate Heptahydrate as a Catalyst. J. Serb. Chem. Soc. 2011, 76, 1191-1198.

(25) Alinezhad, H.; Tajbakhsh, M.; Salehian, F.; Biparva, P. Synthesis of Quinoxaline Derivatives Using $\mathrm{TiO}_{2}$ Nanoparticles as an Efficient and Recyclable Catalyst. Bull. Korean Chem. Soc. 2011, 32, $3720-3725$.

(26) Kumbhar, A.; Kamble, S.; Barge, M.; Rashinkar, G.; Salunkhe, R. Brønsted Acid Hydrotrope Combined Catalyst for Environmentally Benign Synthesis of Quinoxalines and Pyrido[2,3-b]pyrazines in Aqueous Medium. Tetrahedron Lett. 2012, 53, 27562760.

(27) Hasaninejad, A.; Shekouhy, M.; Zare, A. Silica nanoparticles Efficiently Catalyzed Synthesis of Quinolines and Quinoxalines. Catal. Sci. Technol. 2012, 2, 201-214.

(28) Karami, B.; Khodabakhshi, S.; Nikrooz, M. A Modified Synthesis of Some Novel Polycyclic Aromatic Phenazines and Quinoxalines by Using the Tungstate Sulfuric Acid (TSA) as a Reusable Catalyst Under Solvent-free Conditions. J. Chin. Chem. Soc. 2012, 59, 187-192.

(29) Karami, B.; Rooydel, R.; Khodabakhshi, S. A Rapid Synthesis of Some 1,4-aryldiazines by the Use of Lithium Chloride as an Effective Catalyst. Acta Chim. Slov. 2012, 59, 183-188.

(30) Khodabakhshi, S.; Karami, B. A Rapid and Eco-friendly Synthesis of Novel and Known Benzopyrazines Using Silica Tungstic Acid (STA) as a New and Recyclable Catalyst. Catal. Sci. Technol. 2012, 2, 1940-1944.

(31) Soleymani, R.; Niakan, N.; Tayeb, S.; Hakimi, S. Synthesis of Novel Aryl Quinoxaline Derivatives by New Catalytic Methods. Orient. J. Chem. 2012, 28, 687-701.

(32) Habibzadeh, S. 1,3-Dibromo-5,5-dimethylhydantoin (DBH): A Novel and Efficient Catalyst for the Synthesis of Quinoxaline Derivatives Under Solvent-free Conditions. Org. Chem.: Indian J. 2012, 8, 473-476.

(33) Sajjadifar, S.; Zolfigol, M. A.; Mirshokraie, S. A.; Miri, S.; Louie, O.; Nezhad, E. R.; Karimian, S.; Darvishi, G.; Donyadari, E.; Farahmand, S. Facile Method of Quinoxaline Synthesis Using Phenol as a New, Efficient and Cheap Catalyst at Room Temperature. Am. J. Org. Chem. 2012, 2, 97-104.

(34) Kadam, H. K.; Khan, S.; Kunkalkar, R. A.; Tilve, S. G. Graphite Catalyzed Green Synthesis of Quinoxalines. Tetrahedron Lett. 2013, 54, 1003-1007.

(35) Shirini, F.; Akbari-Dadamahaleh, S.; Mohammad-Khah, A.; Aliakbar, A.-R. Rice Husk: A Mild, Efficient, Green and Recyclable Catalyst for the Synthesis of 12-Aryl-8,9,10,12-tetrahydro[a]xanthene11-ones and Quinoxaline Derivatives. C. R. Chim. 2013, 16, 207-216.

(36) Jafarpour, M.; Rezaeifard, A.; Ghahramaninezhad, M.; Tabibi, T. Reusable $\alpha-\mathrm{MoO}_{3}$ Nanobelts Catalyzes the Green and Heterogeneous Condensation of 1,2-Diamines with Carbonyl Compounds. New I. Chem. 2013, 37, 2087-2095.

(37) Mulik, A.; Chandam, D.; Patil, P.; Patil, D.; Jagdale, S.; Deshmukh, M. Proficient Synthesis of Quinoxaline and Phthalazinetrione Derivatives Using [ $\left.\mathrm{C}_{8} \mathrm{dabco}\right] \mathrm{Br}$ Ionic Liquid as Catalyst in Aqueous Media. J. Mol. Liq. 2013, 179, 104-109.

(38) Vahdat, S. M.; Baghery, S. Sulfonated Organic Salts: Recyclable Green Catalysts for the Facile and Rapid Route Synthesis of 2,3Dissubstituted Quinoxaline Derivatives in Water. World Appl. Sci. J. 2013, 21, 394-401.

(39) Karami, B.; Khodabakhshi, S. A Novel and Simple Synthesis of Some New and Known Dibenzo Phenazine and Quinoxaline Derivatives Using Lead Dichloride. J. Chil. Chem. Soc. 2013, 58, 1655-1658.

(40) Vahdat, S. M.; Baghery, S. A Green and Efficient Protocol for the Synthesis of Quinoxaline, Benzoxazole and Benzimidazole Derivatives Using Heteropolyanion-Based Ionic Liquids: As a 
Recyclable Solid Catalyst. Comb. Chem. High Throughput Screening 2013, 16, 618-627.

(41) Sajjadifar, S.; Zolfigol, M. A.; Chehardoli, G.; Miri, S.; Moosavi, P. Qinoxaline II. A Practical Efficient and Rapid Synthesis of New Quinoxalines Catalyzed by Citric Acid as a Trifunctional Brønsted Acid at Room Temperature Under Green Condition. Int. J. ChemTech Res. 2013, 5, 422-429.

(42) Sadeghi, B.; Karimi, F. ZnO Nanoparticles as an Efficient and Reusable Catalyst for Synthesis of Quinoxaline Under Solvent Free Condition. Iran. J. Catal. 2013, 3, 1-7.

(43) Jafarpour, M.; Rezapour, E.; Ghahramaninezhad, M.; Rezaeifard, A. A Novel Protocol for Selective Synthesis of Monoclinic Zirconia Nanoparticles as a Heterogeneous Catalyst for Condensation of 1,2-Diamines with 1,2-Dicarbonyl Compounds. New J. Chem. 2014, $38,676-682$.

(44) Zhang, K.; Dai, Y.; Zhang, X.; Xiao, Y. Synthesis and Photophysical Properties of Three Ladder-type Chromophores with Large and Rigid Conjugation Structures. Dyes Pigm. 2014, 102, 1-5.

(45) Jafari, F.; Khodabakhshi, S. $\mathrm{MnSO}_{4} \cdot \mathrm{H}_{2} \mathrm{O}$ : A Highly Efficient and Inexpensive Catalyst for the Synthesis of Benzo-2-pyrones and Benzopyrazines. Bulg. Chem. Commun. 2014, 46, 36-42.

(46) Sajjadifar, S.; Nezhad, E. R.; Khosravani, E. Synthesis of Quinoxaline Derivatives Using Sulfonic Acid Functionalized Imidazolium Salts as Highly Efficient and Reusable Brønsted Acidic Ionic Liquids Catalysts Under Solvent-free Conditions. Chem. Sci. Trans. 2014, 3, 292-302.

(47) Samanta, S.; das Gupta, A.; Mallik, A. K. An Expedient "Onwater" Synthesis of Quinoxalines. Monatsh. Chem. 2014, 145, 16691673.

(48) Tajbakhsh, M.; Bazzar, M.; Ramzanian, S. F.; Tajbakhsh, M. Sulfonated Nanoclay Minerals as a Recyclable Eco-friendly Catalyst for the Synthesis of Quinoxaline Derivatives in Green Media. Appl. Clay Sci. 2014, 88-89, 178-185.

(49) Jafarpour, M.; Gorzin, G.; Rezaeifard, A. Green Condensation of Various 1,2-diamine and 1,2-dicarbonyl Compounds Catalyzed by Reusable Zirconium (IV) Tetradentate Schiff Base Complex. Curr. Catal. 2014, 3, 260-265.

(50) Sajjadifar, S.; Miri, S. Green Synthesis of Quinoxaline Derivatives Using Phthalic Acid as Difunctional Brønsted Acid at Room Temperature. Int. J. ChemTech Res. 2014, 6, 5433-5440.

(51) Carlier, L.; Baron, M.; Chamayou, A.; Couarraze, G. Greener Pharmacy Using Solvent-free Synthesis: Investigation of the Mechanism in the Case of Dibenzophenazine. Powder Technol. 2013, 240, 41-47.

(52) de Oliveira, P. F. M.; Haruta, N.; Chamayou, A.; Guidetti, B.; Baltas, M.; Tanaka, K.; Sato, T.; Baron, M. Comprehensive Experimental Investigation of Mechanically Induced 1,4-Diazines Synthesis in Solid State. Tetrahedron 2017, 73, 2305-2310.

(53) Haruta, N.; Sato, T.; Tanaka, K.; Baron, M. Reaction Mechanism in the Mechanochemical Synthesis of Dibenzophenazine: Application of Vibronic Coupling Density Analysis. Tetrahedron Lett. 2013, 54, 5920-5923.

(54) Sato, T.; Tokunaga, K.; Tanaka, K. Vibronic Coupling in Naphthalene Anion: Vibronic Coupling Density Analysis for Totally Symmetric Vibrational Modes. J. Phys. Chem. A 2008, 112, 758-767.

(55) Sato, T.; Tokunaga, K.; Iwahara, N.; Shizu, K.; Tanaka, K. In The Jahn-Teller Effect: Fundamentals and Implications for Physics and Chemistry; Köppel, H., Yarkony, D. R., Barentzen, H., Eds.; Springer Series in Chemical Physics; Springer-Verlag: Berlin, 2009; Vol. 97, pp 99-129.

(56) Fukui, K.; Yonezawa, T.; Shingu, H. A Molecular Orbital Theory of Reactivity in Aromatic Hydrocarbons. J. Chem. Phys. 1952, 20, 722-725.

(57) Fukui, K. Recognition of Stereochemical Paths by Orbital Interaction. Acc. Chem. Res. 1971, 4, 57-64.

(58) Parr, R. G.; Yang, W. Density Functional Approach to the Frontier-electron Theory of Chemical Reactivity. J. Am. Chem. Soc. 1984, 106, 4049-4050.
(59) Geerlings, P.; de Proft, F.; Langenaeker, W. Conceptual Density Functional Theory. Chem. Rev. 2003, 103, 1793-1874.

(60) Sato, T.; Iwahara, N.; Haruta, N.; Tanaka, K. C 60 Bearing Ethylene Moieties. Chem. Phys. Lett. 2012, 531, 257-260.

(61) Haruta, N.; Sato, T.; Tanaka, K. Chemical Reactivity in Nucleophilic Cycloaddition to $\mathrm{C}_{70}$ : Vibronic Coupling Density and Vibronic Coupling Constants as Reactivity Indices. J. Org. Chem. 2012, 77, 9702-9706.

(62) Haruta, N.; Sato, T.; Iwahara, N.; Tanaka, K. Vibronic Couplings in Cycloadditions to Fullerenes. J. Phys.: Conf. Ser. 2013, $428,012003$.

(63) Sato, T.; Uejima, M.; Iwahara, N.; Haruta, N.; Shizu, K.; Tanaka, K. Vibronic Coupling Density and Related Concepts. J. Phys.: Conf. Ser. 2013, 428, 012010.

(64) Haruta, N.; Sato, T.; Tanaka, K. Regioselectivity in Multiple Cycloadditions to Fullerene $\mathrm{C}_{60}$ : Vibronic Coupling Density Analysis. Tetrahedron 2014, 70, 3510-3513.

(65) Haruta, N.; Sato, T.; Tanaka, K. Reactivity of Endohedral Metallofullerene $\mathrm{La}_{2} @ \mathrm{C}_{80}$ in Nucleophilic and Electrophilic Attacks: Vibronic Coupling Density Approach. J. Org. Chem. 2015, 80, 141147.

(66) Haruta, N.; Sato, T.; Tanaka, K. Reactivity Index for DielsAlder Cycloadditions to Large Polycyclic Aromatic Hydrocarbons Using Vibronic Coupling Density. Tetrahedron Lett. 2015, 56, 590594.

(67) Frisch, M. J.; Trucks, G. W.; Schlegel, H. B.; Scuseria, G. E.; Robb, M. A.; Cheeseman, J. R.; Scalmani, G.; Barone, V.; Mennucci, B.; Petersson, G. A.; et al. Gaussian 09, Revision C.01; Gaussian Inc.: Wallingford, CT, 2009. 